\title{
Mapping Perceptual Texture Similarity for Image Retrieval
}

\author{
Janet S. Payne ${ }^{1}$ and John Stonham ${ }^{2}$ \\ ${ }^{1}$ Centre for Applied Computing, \\ Faculty of Technology, \\ Buckinghamshire Chilterns University College, \\ High Wycombe, HP11 2JZ, UK \\ janet.payne@bcuc.ac.uk \\ http: / / www . bcuc.ac.uk/ \\ ${ }^{2}$ Dept of Electronic and Computer Engineering, \\ Brunel University, \\ Uxbridge, UB8 3PH \\ john. stonham@brunel.ac.uk \\ http: / /www.brunel.ac.uk/
}

\begin{abstract}
Images are being produced and made available in ever increasing numbers; but how can we find images "like this one" that are of interest to us? Many different systems have been developed which offer content-based image retrieval (CBIR), using low-level features such as colour, texture and shape; but how can the retrieval performance of such systems be measured? We have produced a perceptually-derived ranking of similar images using the Brodatz textures image dataset, based on a human study, which can be used to benchmark retrieval performance. In this paper, we show how a "mental map" may be derived from individual judgements to provide a scale of psychological distance, and a visual indication of image similarity.
\end{abstract}

\section{Measuring the Effectiveness of Image Retrieval}

Digital images are being produced at a greater rate than ever before; digital cameras and cameraphones are widely available, and widely used. Art collections such as St Petersburg's Hermitage Museum or London's National Gallery, and photographic archives, continue to be digitised and made available via the Web. But how can we find images of interest to us among the petabytes of visual data? We have become used to efficient retrieval of text and web pages using keywords; but image retrieval remains a difficult task, in spite of the many research efforts applied over the past decade or more. Images for professional and technical uses, such as medical data, have standards that specify the metadata to be attached to each image [3]; however, the original annotator is unlikely to have anticipated all future potential uses of the images. Pictures taken for personal interest may also be of interest to others, both at the time, and for historical reasons in the future; experience shows that these are even less likely to have any kind of meaningful metadata [2], as a glance at the "properties" of any document file will demonstrate. Content-based image retrieval (CBIR) appears to be 
essential, using low-level image features rather than relying on annotation to locate more images "like this one". Colour, texture and shape have all been used for this purpose, from IBM's QBIC onwards [7]; texture in particular has been used successfully for many different applications $[3,11]$.

\subsection{How Should Similarity Be Judged for Images?}

But how should image similarity be judged? Various measures for similarity of images have been proposed; these are typically based on distance measures in psychological space. Euclidean distance is perhaps the most widely used. However, Tversky (1977) criticizes the use of standard metrics, pointing out that similarity is often not symmetric [12]. As he points out, "a is like b" is generally not equivalent to "b is like a"; the more salient stimulus tends to be selected as the prototype or referent. Among the examples he used, geometric figures were considered to be more salient the better the "form" (regularity of outline); and where two figures were similar for this, the more complex one was considered to be more salient. We observed this asymmetry of similarity in this research, as figures 4 and 5 show.

Surely CBIR is intended for use by people interested in what the images resemble, rather than in learning a specialized database query language or an esoteric coding scheme. It seems appropriate to search on what the images actually "look like" to the potential users of such systems. In order to compare the performance of different CBIR systems, some standard image dataset is required. For texture, the most widely used one is still the Brodatz photographic album "Textures" [1], as used for example by Nunes et al [8]. Some example tiles from the Brodatz album are shown in fig. 1. Typically, a retrieval technique is judged by how many other tiles of the same texture it returns when presented with a tile from each texture as a query image [6]. This fails to take into account images which are not homogenous, nor does it consider different textures which the human observer would consider to be similar. In our research, we have developed a perceptually-derived ranking of similar images from the Brodatz dataset which can be used to evaluate retrieval performance [10]. Earlier studies of perceptual similarity have tended to use only a subset of the Brodatz textures; we have used the full set of 112 images.

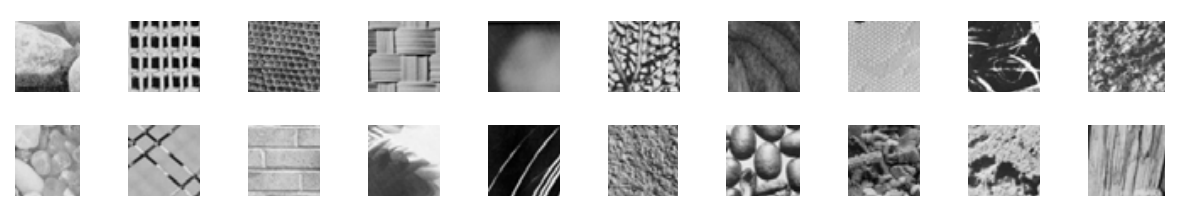

Fig. 1. Examples from the Brodatz album

\section{Texture Similarity Derived from Perceptual Judgements}

The participants in our study were asked to select up to four images, in order, which they considered most like each of the full set of the 112 Brodatz images, viewed on a 
computer screen [9]. The display showed 15 textures, arranged as three rows of five images, with the query image placed in the middle. Thirty people took part in this study, aged between 18 and 54, with a median age of 26; ten were female, twenty male. They included an art historian, a chemist, several computing students, a physicist, a psychology student, and a zoologist. It was noted that individuals tended to agree on similarity for regular textures such as reptile skin (eg D3, or D22), or pebbles (eg D30), or fine textures such as D4. Irregular textures such as D62, or D87, showed less agreement. Although individuals were free to select up to four "most like" images from the set displayed on the screen, it would have been surprising if there had been no agreement between them. Correlation measures this, and can be used to calculate the principal components of the responses.

For example, consider the selections made for D30 (table 1): a value of $\mathbf{5}$ is assigned to the query image, $\mathbf{4}$ to each individual's first choice, $\mathbf{3}$ to their second choice, and so on. In this case, only seven other images out of the possible fourteen were considered to be similar by the participants. Most people made four choices, but not everyone did; for example, the second participant made only one selection.

Table 1. Selections for D30 as query, by participant

\begin{tabular}{|r|l|l|l|l|l|l|l|l|l|l|l|l|l|l|l|l|l|l|l|l|l|l|l|l|l|l|l|l|l|l|}
\hline D23 & 3 & 0 & 3 & 3 & 3 & 3 & 4 & 3 & 4 & 3 & 3 & 3 & 3 & 3 & 3 & 3 & 3 & 3 & 1 & 2 & 3 & 4 & 0 & 2 & 1 & 4 & 3 & 0 & 2 & 3 \\
\hline D27 & 1 & 0 & 1 & 0 & 2 & 1 & 2 & 1 & 1 & 1 & 0 & 1 & 0 & 0 & 0 & 0 & 1 & 1 & 3 & 0 & 1 & 2 & 0 & 1 & 2 & 1 & 1 & 0 & 1 & 2 \\
\hline Query & 5 & 5 & 5 & 5 & 5 & 5 & 5 & 5 & 5 & 5 & 5 & 5 & 5 & 5 & 5 & 5 & 5 & 5 & 5 & 5 & 5 & 5 & 5 & 5 & 5 & 5 & 5 & 5 & 5 & 5 \\
\hline D31 & 4 & 4 & 4 & 4 & 4 & 4 & 3 & 4 & 2 & 4 & 4 & 4 & 4 & 4 & 4 & 4 & 4 & 2 & 4 & 4 & 4 & 3 & 4 & 4 & 4 & 2 & 4 & 4 & 4 & 4 \\
\hline D62 & 0 & 0 & 0 & 0 & 0 & 2 & 0 & 0 & 0 & 0 & 0 & 0 & 1 & 0 & 0 & 0 & 0 & 0 & 0 & 1 & 0 & 0 & 3 & 3 & 0 & 0 & 0 & 2 & 0 & 1 \\
\hline D66 & 0 & 0 & 0 & 0 & 0 & 0 & 1 & 0 & 0 & 0 & 1 & 0 & 0 & 0 & 0 & 0 & 0 & 4 & 0 & 0 & 0 & 0 & 0 & 0 & 0 & 0 & 0 & 0 & 0 & 0 \\
\hline D98 & 2 & 0 & 2 & 2 & 1 & 0 & 0 & 2 & 3 & 2 & 2 & 2 & 2 & 2 & 2 & 2 & 2 & 0 & 2 & 3 & 2 & 1 & 0 & 0 & 3 & 3 & 2 & 1 & 3 & 0 \\
\hline D99 & 0 & 0 & 0 & 0 & 0 & 0 & 0 & 0 & 0 & 0 & 0 & 0 & 0 & 1 & 1 & 0 & 0 & 0 & 0 & 0 & 0 & 0 & 1 & 0 & 0 & 0 & 0 & 3 & 0 & 0 \\
\hline
\end{tabular}

\subsection{Principal Components of the Perceptual Ranking}

Principal components analysis (PCA), also known as the Karhunen-Loéve transform, is a form of data reduction; a mathematical procedure for transforming a number of (possibly) uncorrelated variables into a smaller number of uncorrelated variables, known as principal components [5]. The first principal component accounts for as much of the variability in the data as possible, and each following one for as much of the remaining variability as possible. Each component is orthogonal to the others; it can be considered as a translation, followed by a rotation, so that the data fits better around the axes, bringing out underlying patterns in the data.

Starting from the correlation matrix of the data, the eigenvectors (which correspond to the principal components) for the $n$ biggest eigenvalues (the variances) are selected and transformed. The number of eigenvalues required to preserve most of the variance can be obtained from the shape of the "knee" in a plot of sorted normalised eigenvalues, known as a "scree diagram", from its shape (eg as in fig. 2, for D30, pebbles). 


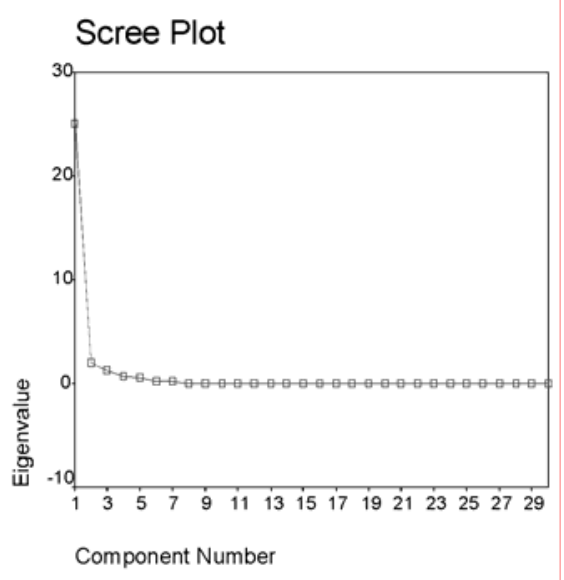

Fig. 2. Scree plot for PCA extraction, texture D30

The table below (Table 2) gives the PCA components for texture D30, shown to two decimal places: three components were extracted; the scree plot indicates that two were sufficient. "Subject" refers to the participants, listed alphabetically by their initials in the same order as in Table 1 above. For this texture, the $1^{\text {st }}$ component accounts for $83.5 \%$ of the variance. In almost all cases for the full Brodatz image set and the selections made by the participants in our research, the $1^{\text {st }}$ component accounts for over $90 \%$ of the variance.

Table 2. PCA components, for D30, alpabetical by participant

\begin{tabular}{|c|c|c|c|}
\cline { 2 - 4 } \multicolumn{1}{c|}{} & \multicolumn{3}{c|}{ Component } \\
\hline Subject & $\mathbf{1}$ & $\mathbf{2}$ & $\mathbf{3}$ \\
\hline AH & 0.99 & -0.09 & -0.05 \\
\hline AT & 0.88 & 0.40 & 0.04 \\
\hline AW & 0.99 & -0.09 & -0.05 \\
\hline BH & 0.98 & -0.05 & -0.08 \\
\hline BT & 0.96 & -0.05 & 0.15 \\
\hline CD & 0.89 & 0.24 & 0.31 \\
\hline ER & 0.86 & -0.20 & 0.45 \\
\hline FB & 0.99 & -0.09 & -0.05 \\
\hline GS & 0.87 & -0.41 & -0.10 \\
\hline HF & 0.99 & -0.09 & -0.05 \\
\hline JG & 0.96 & -0.08 & -0.02 \\
\hline JP & 0.99 & -0.09 & -0.05 \\
\hline KM & 0.97 & 0.04 & -0.06 \\
\hline LT & 0.96 & -0.01 & -0.12 \\
\hline MD & 0.96 & -0.01 & -0.12 \\
\hline
\end{tabular}

\begin{tabular}{|c|r|r|r|}
\cline { 2 - 4 } \multicolumn{1}{c|}{} & \multicolumn{3}{c|}{ Component } \\
\hline Subject & $\mathbf{1}$ & $\mathbf{2}$ & $\mathbf{3}$ \\
\hline MG & 0.98 & -0.05 & -0.08 \\
\hline MI & 0.99 & -0.09 & -0.05 \\
\hline MM & 0.58 & -0.20 & 0.58 \\
\hline MT & 0.85 & 0.09 & -0.09 \\
\hline PB & 0.93 & 0.10 & -0.28 \\
\hline PH & 0.99 & -0.09 & -0.05 \\
\hline RA & 0.92 & -0.24 & 0.23 \\
\hline RD & 0.66 & 0.73 & 0.08 \\
\hline SF & 0.80 & 0.45 & 0.28 \\
\hline SH & 0.88 & 0.05 & -0.29 \\
\hline SM & 0.87 & -0.41 & -0.10 \\
\hline SP & 0.99 & -0.09 & -0.05 \\
\hline ST & 0.62 & 0.70 & -0.17 \\
\hline TC & 0.95 & -0.03 & -0.27 \\
\hline WH & 0.91 & 0.11 & 0.33 \\
\hline
\end{tabular}




\section{A Mental Map of Perceived Similarity}

A consensus view can be obtained by applying the first principal component to the matrix representing individual participants' selections; for example, subjects $\mathrm{AH}, \mathrm{AW}$, FB, HF, JP, MI, PH and SP all contributed 0.99 to this viewpoint, while MM was only 0.58 . Multiplying out the responses gives the following values, shown to four decimal places, in Table 3. D31 has a value very close to that of the query image, D30; as Table 1 shows, all but five of the participants selected it as most like the query. D66 and D99 were only selected by three or four people respectively; the computed value is much lower.

Table 3. Computed values for D30

\begin{tabular}{|c|c|}
\hline D23 & 72.5250 \\
\hline D27 & 24.5300 \\
\hline Query & 136.0900 \\
\hline D31 & 102.4660 \\
\hline D62 & 10.2140 \\
\hline D66 & 4.1500 \\
\hline D98 & 45.2670 \\
\hline D99 & 4.4460 \\
\hline
\end{tabular}

Scaling to a range of 0 for the query, 1 for the least like image, produces a scaled similarity vector (Table 4 , shown to four decimal places).

Table 4. Scaled similarity for D30, from $1^{\text {st }}$ principal component

\begin{tabular}{|l|r|r|r|r|r|r|r|}
\hline D30 & D31 & D23 & D98 & D27 & D62 & D99 & D66 \\
\hline 0 & 0.2548 & 0.4818 & 0.6884 & 0.8455 & 0.9540 & 0.9978 & 1 \\
\hline
\end{tabular}

Gould and White (1986) show how individual ranking judgements can be transformed into such composite similarity scales [4]. Their examples are drawn from geography, and allow factors such as perceived attractiveness of different towns or regions of a country to be plotted, overlaid on a cartographic map; they call this approach "Mental Mapping". The same technique can be applied to the perceptually ranked images in our research. Using the scaled values of Table 4, a "mental map" can now be plotted, showing the ordering and relative spacing of the images; a distance measure has been derived corresponding to perceptual similarity. Fig. 3 indicates the relative distances, using the scaled values of Table 4 , at 0.1 intervals, to provide a visual indication of the perceptually-derived ranking for this texture. 


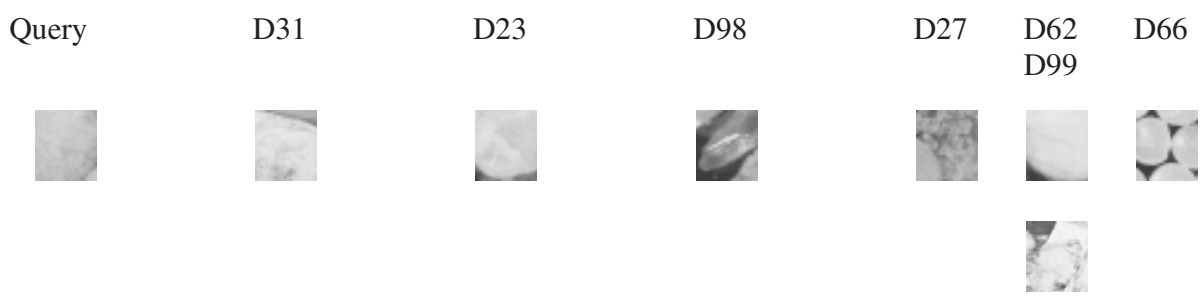

\begin{tabular}{|l|l|l|l|l|l|l|l|l|l|l|}
\hline 0 & 0.1 & 0.2 & 0.3 & 0.4 & 0.5 & 0.6 & 0.7 & 0.8 & 0.9 & 1 \\
\hline
\end{tabular}

Fig. 3. Images along similarity scale, for D30

\subsection{Similarity Is not Always Symmetric}

Texture D1 is one of the easily identifiable images, of wire netting. However, although there are two other images, D46 and D47, also of wire netting, neither have the same orientation. The scaled similarity (table 5) shows that no other image was considered a close match; figure 4 plots the selected images along a scale from 0 to 1 .

Table 5. Scaled similarity, from $1^{\text {st }}$ principal component for D1

\begin{tabular}{|c|c|c|c|c|c|c|c|c|c|c|}
\hline D1 & D64 & D20 & D65 & D95 & D8 & D82 & D104 & D47 & D35 & D80 \\
\hline 0 & 0.6809 & 0.6970 & 0.7385 & 0.7658 & 0.7991 & 0.8418 & 0.9382 & 0.9939 & 0.9940 & 1 \\
\hline
\end{tabular}

D1

$\begin{array}{rrrrr}\text { D64 } & \text { D65 } & & \text { D104 } & \\ \text { D20 } & \text { D95 } & & \text { D47 } & \text { D80 }\end{array}$
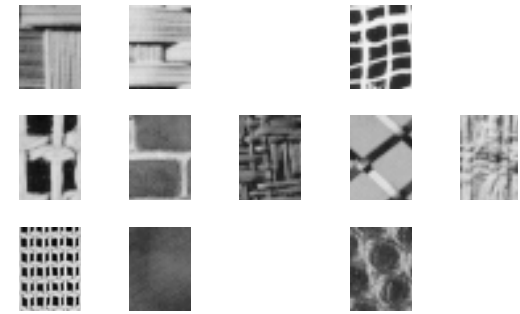

\begin{tabular}{|l|l|l|l|l|l|l|l|l|l|l|}
\hline 0 & 0.1 & 0.2 & 0.3 & 0.4 & 0.5 & 0.6 & 0.7 & 0.8 & 0.9 & 1 \\
\hline
\end{tabular}

Fig. 4. Images along similarity scale, for D1

The closest comparisons are to D64, D20, D65, D95, and D8, all of which show the same orientations at a similar scale. The next group includes D47, one of the other wire images, but this was considered less good a match. D46, the back-lit wire image, was not selected by any of the participants as a match for D1.

If D47 is next considered (table 6 and fig. 5), the lack of symmetry in perceptual similarity [12] is very noticeable. Here, the diagonal orientation is the most salient 
feature, shown by the closeness of D46, then of D18. The remaining textures match on scale, or on grey level; D1, the only other image of wire, is not considered a match.

Table 6. Scaled similarity, from $1^{\text {st }}$ PCA component for D47

\begin{tabular}{|c|c|c|c|c|c|c|c|c|c|}
\hline D47 & D46 & D18 & D101 & D52 & D35 & D102 & D16 & D17 & D50 \\
\hline 0 & 0.2827 & 0.4994 & 0.8481 & 0.8593 & 0.9383 & 0.9583 & 0.9633 & 0.9763 & 1 \\
\hline
\end{tabular}

D46

D18

D101 D102

D52 D16

D50

D17
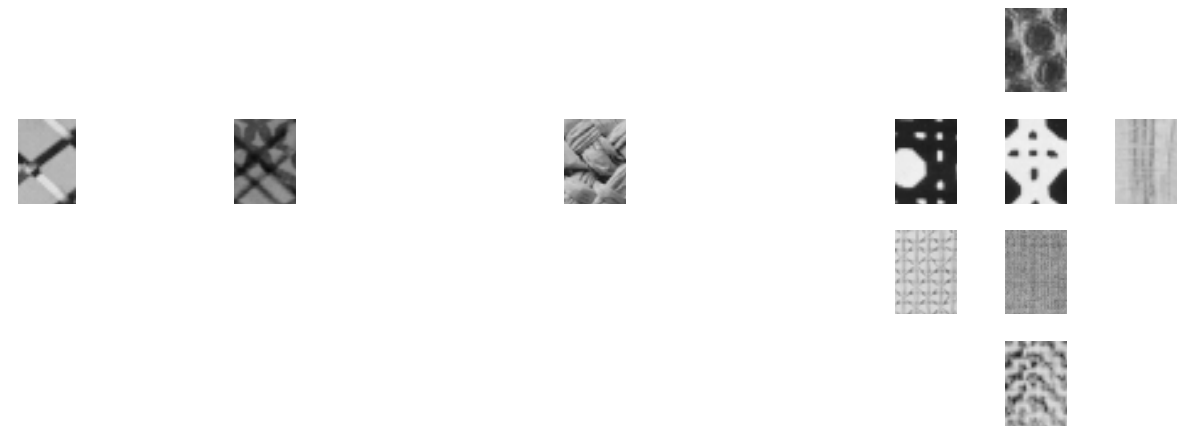

\begin{tabular}{|l|l|l|l|l|l|l|l|l|l|l|}
\hline 0 & 0.1 & 0.2 & 0.3 & 0.4 & 0.5 & 0.6 & 0.7 & 0.8 & 0.9 & 1 \\
\hline
\end{tabular}

Fig. 5. Images along similarity scale, for D47

\subsection{Salient Features}

The reptile skin textures (D3, D22, D35 and D36) are very similar in regularity, and orientation (Table 7, and Fig 6). Taking D3 as the query image, the scale increases from D36, the most like, to D35, considered least like within this group.

Table 7. Scaled similarity, for D3

\begin{tabular}{|l|l|l|l|l|l|l|}
\hline D3 & D36 & D22 & D35 & D104 & D84 & D24 \\
\hline 0 & 0.2785 & 0.4161 & 0.6221 & 0.8885 & 0.9195 & 1 \\
\hline
\end{tabular}

This demonstrates that human observers are not scale invariant, unlike some of the techniques proposed for image retrieval. Small scale is considered more salient here. 


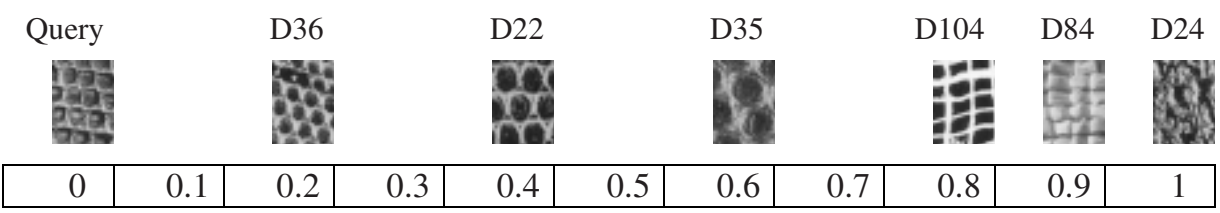

Fig. 6. Images along similarity scale, for D3

Considering now two of the "brick wall" textures, D26 and D94 (Table 9). D26 is considered to be most similar to D94, and vice versa, but the psychological distance between each as the query and the remaining brick textures, D25, D95 and D96, is noticeably different in each case.

Table 9. Scaled similarity, for brick patterns D26, and D94

\begin{tabular}{|c|c|c|c|c|c|c|}
\hline D26 & D94 & D25 & D95 & D96 & D64 & D50 \\
\hline 0 & 0.2856 & 0.6277 & 0.6706 & 0.6953 & 0.8682 & 1 \\
\hline D94 & D26 & D95 & D25 & D96 & D64 & D56 \\
\hline 0 & 0.2913 & 0.5917 & 0.8221 & 0.9030 & 0.9414 & 1 \\
\hline
\end{tabular}

Figures 8 and 9 show this. Here, grey level appears to be the most salient feature, hence the closeness of D26 and D94 to each other. After this, scale applies, as it did for the reptile skin examples, all of which were of the same grey level and contrast.

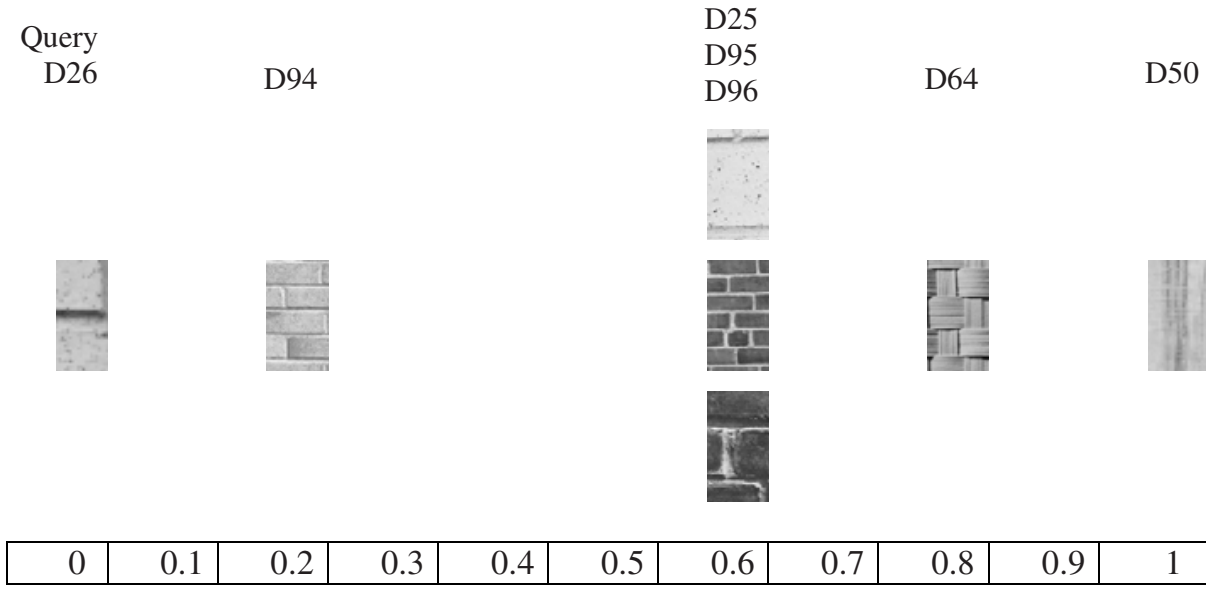

Fig. 8. Images along similarity scale, for D26

D25 is considered to be similar to D26, and D95 to D94. Since Brodatz deliberately organised his photographs of textures to bring out similarities and contrasts, it would be expected that the participants in our study reached a similar ordering to his. 


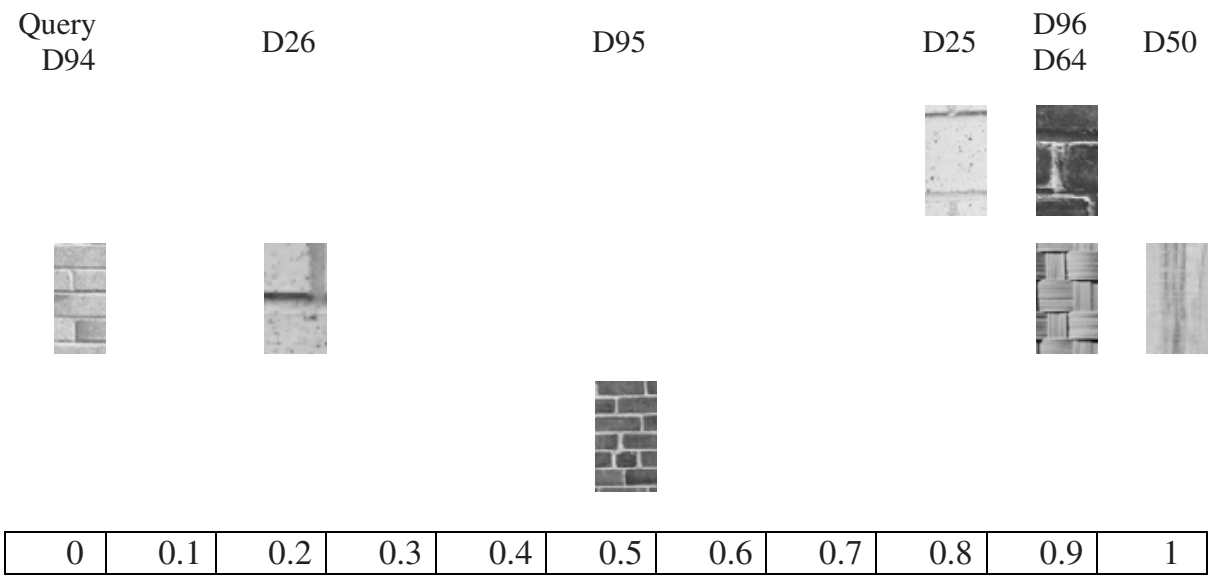

Fig. 9. Images along similarity scale, for D94

\section{Conclusions}

Images and searching for images continues to be an area of interest; individuals tend not to add metadata to their photographs; and even if they do, it is unlikely that the annotator will have considered all possible future uses of each image. Low-level features of the image itself can always be used, since they are an intrinsic part of the data itself; texture is particularly suitable, and applies equally to colour or "monochrome" originals.

However, if an image retrieval system is designed for end-users, rather than for specialists, it is unreasonable to expect such end-users to learn a specialised query language or interface; it is surely preferable to evaluate the retrieval performance of CBIR systems using perceptual judgements of image similarity. This research has shown how such a benchmark set may be obtained, working with the widely-used "Brodatz" textures image dataset.

We have shown how Gould and White's method of "mental mapping" may be applied to the individual perceptual ranking judgements. Correlation coefficients are calculated between each individual's ranking for each of the 112 Brodatz textures in turn; principal components analysis (PCA) is then applied to the correlation matrix for each texture. We found that the $1^{\text {st }}$ principal component accounts for over $90 \%$ of the variance in almost all cases; for the example used in this paper, it accounted for $83.5 \%$. This $1^{\text {st }}$ principal component represents the weighting of each individual's view to the consensus view; multiplying out the response matrix with the $1^{\text {st }}$ principal component and scaling the result, indicates psychological distance between similar images.

It was also observed that image similarity is not always symmetric, and that features such as scale, orientation, and contrast, affect the perceived saliency of such images. 


\section{References}

1. P. Brodatz, Textures - A Photographic Album For Artists And Designers, Dover (New York, 1966)

2. D.C.A. Bulterman, Is It Time for a Moratorium on Metadata?, IEEE Multimedia, Oct/Dec 2004, Vol 11, pp10-17

3. T. Glatard, J. Montagnat, I. E. Magnin, Texture Based Medical Image Indexing and Retrieval: Application to Cardiac Imaging, Proceedings 6th ACM SIGMM International Workshop on Multimedia Image Retrieval (MIR 2004), New York, October 15-16, 2004, pp135-142

4. P. Gould and R. White, Mental Maps (2nd ed), Routledge (London, 1986, reprinted 2002)

5. I. T. Jolliffe, Principal Component Analysis (2nd ed), Springer (New York, 2002)

6. B. S. Manjunath and W. Y. Ma, Texture Features for Browsing and Retrieval of Image Data, In: IEEE Transactions on Pattern Analysis and Machine Intelligence, Aug 1996, Vol 18 , No 8, pp837-842

7. W. Niblack, R. Barber, W. Equitz, M. Flickner, E. Glasman, D. Petkovic and P. Yanker, The QBIC Project: Querying Images By Content Using Color, Texture \& Shape, IBM Research Report RJ9203, 1 Feb 1993.

8. J. C. Nunes, O. Niang, Y. Bouaoune, E. Delechelle, and P. Bunel, Bidimensional Empirical Mode Decomposition Modified for Texture Analysis, In: Proceedings of the 13th Scandinavian Conference on Image Analysis, Göteborg, Sweden, June 2003, pp. 171-177.

9. J. S. Payne, L. Hepplewhite and T. J. Stonham, Evaluating Content-Based Image Retrieval Techniques Using Perceptually Based Metrics, In: Proceedings of SPIE Electronic Imaging, San Jose, USA, Jan 1999, vol. 3647, pp. 122-133.

10. J. S. Payne and T. J. Stonham, Can Texture and Image Content Retrieval Methods Match Human Perception?, In: International Symposium on Intelligent Multimedia, Video and Speech Processing (ISIMP'2001), Hong Kong, May 2001, pp. 154-157.

11. M. Pietikäinen, T. Ojala and O. Silven, Approaches to Texture-based Classification, Segmentation and Surface Inspection, In: Handbook of Pattern Recognition and Computer Vision (2nd ed), 1998, pp. 711-736.

12. A. Tversky, Features of Similarity, In: Psychological Review, July 1977, Vol 84 No 4, pp. 327-352 